\title{
NIVEL DE SUSTENTABILIDAD EN PEQUEÑAS EXPLOTACIONES SOJERAS DEL CHACO - ARGENTINA. RENTABILIDAD Y ACEPTACIÓN SOCIAL
}

\author{
SUSANA PAULINA GUSINSKY DE GELMAN \\ ANTONIO HUMBERTO CLOSAS \\ ROSA TERESA CRUZ DE INNOCENTE \\ MARGARITA CARLOTA CARBAJAL \\ SUSANA ROSA JEREZ \\ IDALIA GABRIELA DE CASTRO
}

FACULTAD DE CIENCIAS ECONÓMICAS UNIVERSIDAD NACIONAL DEL NORDESTE

ARGENTINA 


\section{RESUMEN}

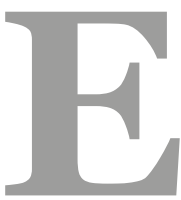

n el Chaco se conjugan, la importancia económica del cultivo de soja con la significación social de las pequeñas empresas agrícolas, predominantemente familiares. Es objetivo del presente trabajo, describir las condiciones de producción de soja evaluando el grado de sustentabilidad de las pequeñas empresas chaqueñas, particularmente: rentabilidad y aceptación social. Las decisiones metodológicas definieron un diseño no experimental, observacional, descriptivo, transversal y prospectivo, cuya recolección de datos se implementó con un cuestionario ad hoc. La muestra está integrada por 27 participantes escogidos en forma aleatoria de una población seleccionada de manera intencional. Los resultados se agrupan conforme las variables del estudio. Vinculado a rentabilidad, indican que el 59.26\% de los productores está disconforme con el beneficio obtenido; un 29,63\% contrata seguros agrícolas para gestionar el riesgo climático; y la comercialización se canaliza principalmente a través de acopiadores locales. El precio se fija teniendo como referencia la operatoria del mercado de cereales de Rosario, neto de costos de transporte e intermediación. El volumen de producción, representativo del rendimiento normal, es $2000 \mathrm{~kg} / \mathrm{ha}$ (valor inferior a la media provincial). Respecto a aceptación social, la mayoría de los agricultores sojeros disponen de 120 hectáreas no propias, su familia no vive ni trabaja en el campo, hecho que impide desarrollar sentido de pertenencia y transferir el saber hacer. La ocupación es de una persona cada cien hectáreas cultivadas. Finalmente, se anhela que éste estudio, contribuya a una explotación sustentable y con la comunidad científico-profesional involucrada.

Palabras clave: soja, agricultura familiar, pequeña empresa.

\section{ABSTRACT}

In Chaco, the economic importance of soybean farming and the social impact of small-scale agricultural businesses, mainly family owned ones, are highly related.

The aim of this study is to describe the soybean production conditions by assessing the sustainability level of small-scale farming businesses in Chaco, especially their profitability and social impact. The research design used was a non-experimental, observational, descriptive, transversal and prospective study which reflects the methodology selected. The data collection method used was an ad hoc questionnaire. From a group deliberately chosen, the sample included 27 participants selected at random.

The results are sorted according to the research variables. As regards profitability, 29.26\% of the farmers are disappointed by the profit obtained; $29.63 \%$ purchase crop insurance coverage 
to manage climate risks; and the commercialization is carried out through local middlemen. The price is set taking into account the Rosario city grain market prices, net cost of transportation and intermediation. The production volume, which indicates the normal production, is of 2000 $\mathrm{kg} / \mathrm{ha}$ (lower than the State average yield). Regarding social impact, most soybean farmers rent $12 \mathrm{ha}$; their families neither live nor work on the farm; this fact prevents them from developing a sense of belonging and from transmitting the know-how. One worker per 100 hectares of cultivated land is the average number of workers in the area.

Finally, we expect that this study would contribute to encourage sustainable production which may include the help of the professional-scientific community.

Keywords: soybeans, family farms, small-scale business.

\section{INTRODUCCIÓN:}

El avance del cultivo de soja en Argentina se vio favorecido por factores internos y externos. Además, en el Chaco, se conjugan la importancia económica del cultivo con la significación social de las pequeñas explotaciones agrícolas, predominantemente familiares. Esto pone de relieve analizar -a nivel microeconómico- en qué condiciones debe llevarse a cabo la producción para que sus efectos netos residuales sean positivos; ya que, pone en funcionamiento un enorme engranaje de servicios, insumos y transporte, pero, ha sido escasa la evaluación de su permanencia en condiciones sustentables.

La delimitación teórica del tema abordado involucra las siguientes definiciones:

1) Explotación sustentable: es aquella que protege la integridad de los recursos naturales, es rentable y socialmente aceptada.

La integridad de los recursos naturales, está ligada a la implementación de "Buenas Prácticas Agrícolas” (Inciarte, 2004) consistentes en la aplicación del conocimiento disponible a la utilización sostenible de los recursos naturales básicos; y la observación de indicadores químicos y físicos asociados con la calidad/salud del suelo.

La rentabilidad se obtiene a partir de la sumatoria algebraica de las causas generadoras de resultado. Los principales elementos a considerar son, sin duda, los ingresos y gastos. Los primeros, dependen de hechos no controlables por el ente (volumen de producción y precio de venta de mercado), en tanto que los últimos tienen comportamientos diferenciados que marcan su incidencia en la ecuación final.

La aceptación social, se vincula con la contribución que la explotación sojera genera para la comunidad en la que está inserta donde se vuelcan sus efectos positivos, vía pago de impuestos, sostenimiento del nivel de empleo y/o desarrollo de la actividad comercial, entre otros. 
SUSANA PAULINA GUSINSKY DE GELMAN - ANTONIO HUMBERTO CLOSAS - ROSA TERESA CRUZ DE INNOCENTE -

MARGARITA CARLOTA CARBAJAL - SUSANA ROSA JEREZ - IDALIA GABRIELA DE CASTRO

NIVEL DE SUSTENTABILIDAD EN PEQUEÑAS EXPLOTACIONES SOJERAS DEL CHACO (ARGENTINA)

Pags. $102-124$

2) Pequeña empresa: cuya descripción comparte características consideradas por Scheinkerman $(2007,2009)$ para "explotación agropecuaria familiar (EAP)" como ser: el trabajo directo y personal del agricultor, no más de dos trabajadores contratados y, determinados limites de extensión y superficie cultivada. En este estudio, está representada por el pequeño productor sojero de la Provincia del Chaco.

Los mayores avances de investigación en soja refieren a la Región Pampeana ${ }^{1}$ (Scheinkerman, 2007, 2009; Casparri y García Fronti, 2011), sin embargo, dichos estudios no son directamente aprovechables para la Provincia del $\mathrm{Chaco}^{2}$ cuyo clima y suelo no presenta las mismas ventajas comparativas que la zona central del país.

Teniendo en cuenta lo mencionado en este punto, la hipótesis sustantiva es la siguiente: "la producción sojera genera efectos socio-económicos positivos, si las pequeñas empresas chaqueñas son explotaciones sustentables”. Estrechamente asociado con la presunción que da validez conceptual a este trabajo, su objetivo consiste en describir las condiciones en las que se lleva a cabo la producción sojera y evaluar el grado de sustentabilidad de las pequeñas empresas del Chaco, en particular lo vinculado a: rentabilidad y aceptación social.

Evaluar la sustentabilidad de las pequeñas explotaciones sojeras del Chaco, implica analizar integralmente las tres variables que la definen. En esta oportunidad el énfasis reside en dos de ellas, dado que lo atinente a la protección de la integridad de los recursos naturales fue examinado en Gusinsky, Cruz, Carbajal, Jerez y de Castro (2013); a cuyo texto puede remitirse el lector interesado.

\section{2) MATERIALES Y MÉTODO}

Este trabajo se desarrolla siguiendo una estructura clásica. Se divide en dos partes, la primera corresponde al análisis teórico; mientras que la segunda al estudio empírico, en cuyo marco se plantean la discusión de los resultados y las conclusiones.

Las características metodológicas se indican seguidamente; contemplando distintos aspectos del diseño y los participantes (población y muestra). El apartado se completa con la descripción de las variables, el instrumento de medida y el procedimiento utilizado para recoger los datos.

\subsection{Diseño de la investigación}

Difícilmente el diseño de una investigación se apoye sólo en métodos puros, más aún cuan-

\footnotetext{
${ }^{1}$ Conformada por las provincias de Buenos Aires, Entre Ríos, Santa Fe, Córdoba, La Pampa y San Luis, considerada la más importante del territorio argentino desde el punto de vista económico, ya que sus características de clima y suelo la transforman en una zona agrícola y ganadera por excelencia. Obtenido el 18-12-2012 en www.mininterior.gov.ar/municipios/gestion/regiones_archivos/Pampeana.pdf

${ }^{2}$ Conjuntamente con Corrientes, Formosa y Misiones integran el Noreste Argentino.
} 
do los criterios (o sus categorías) de selección no son mutuamente excluyentes. Por lo tanto, es común que -el diseño- presente características de un conjunto variado de ambos elementos.

En vista de lo señalado, esta investigación, en función del objetivo que busca, es inicialmente de naturaleza no experimental y observacional; en un segundo momento explicativa. Si se considera como criterio el tipo de información que interesa y el modo de recogerla, es de estilo descriptivo y; en atención a la forma de administrar el instrumento de medición, se empleó la técnica del cuestionario.

A su vez, teniendo en cuenta el marco donde se llevó a cabo, es una investigación de campo. Asimismo, en razón de cómo se miden, analizan y recolectan los datos, este estudio es de línea cuanti-cualitativa y de corte transversal, en tanto que su propósito la hace de tipo prospectivo.

\subsection{Participantes}

Universo: El conjunto de elementos a los cuales se quiere extrapolar los resultados de la investigación se configura con los pequeños productores sojeros chaqueños considerando como tales quienes cumplen con todas las características cuantitativas y cualitativas que a continuación se enuncian:

1) La explotación no supera: quinientas (500) hectáreas de superficie total, doscientas (200) hectáreas cultivadas y, dos (2) empleados no familiares remunerados.

2) Los productores: son personas físicas (empresas unipersonales) que trabajan directamente en su explotación agrícola.

Población: Se estimó pertinente centralizar la investigación empírica, en el espacio geográfico de la subzona XVI-B³ (INTA, 2010), la que, consistente con campañas anteriores, concentra el 55\% (349.500 ha) de la superficie total (639.0oo ha) cultivada con soja en el Chaco (INTA, 2011).

El equipo de investigación definió el criterio para elegir los sujetos que podían facilitar información. Por consiguiente, la selección de la población (en cuanto se refiere al tamaño de empresa y zona geográfica) fue de carácter intencional, dado que incluyó a ochenta y cuatro productores sojeros con explotaciones de hasta 200 hectáreas cultivadas, ubicadas en la sub zona XVI-B, que configuraron la población de este estudio.

Muestra: La realización de un censo que permita estudiar cada uno de los elementos de la citada población fue impracticable, por lo tanto, para la aplicación del instrumento ad hoc, se efectuó una selección probabilística del grupo definitivo de pequeños productores sojeros

\footnotetext{
${ }^{3}$ La Provincia del Chaco está segmentada en cuatro zonas agropecuarias identificadas como XVI: Centro oeste del Chaco, XVII: Ganadera del este, XVIII: Mixta del norte y XIX: Noroeste del Chaco; siendo de particular interés, por su fuerte concentración agrícola, la zona XVI que se divide en tres sub zonas:

* XVI-A: Central, departamentos Comandante Fernández, Independencia, Quitilipi y Maipú.

* XVI-B: del Oeste, con epicentro en la localidad de General Pinedo e integrada por los Departamentos: General Belgrano, 9 de Julio, Chacabuco, 12 de Octubre y 2 de Abril.

* XVI-C: Mixta Sudoeste, departamentos de O’Higgins, San Lorenzo, Fontana, y Santa María de Oro.
} 
chaqueños. Así, la muestra aceptante (Fox, 1981) quedó conformada por un total de veintisiete participantes, escogidos utilizando el método aleatorio simple ya que dentro de la zona seleccionada, todos tuvieron la misma probabilidad de integrarla.

La representatividad se encuentra respaldada por las siguientes características:

1) Contiene todos los departamentos de la zona agrícola que aportó la mayor parte de la superficie sembrada con soja en el Chaco durante el año 2011 y anteriores.

2) La ubicación geográfica de las empresas se realizó a partir de estudios previos realizados por el Instituto Nacional de Tecnología Agropecuaria (INTA).

3) El tamaño muestral es equivalente al $32.14 \%$ de la población, por lo que se presume que el número de observaciones realizadas es razonable.

4) El método probabilístico de extracción de los casos particulares que fueron entrevistados ha consolidado la fortaleza de la representatividad.

Conforme lo expresado sobre población y muestra, y a efectos de sustentar las decisiones metodológicas asumidas se sostiene que:

1) El muestreo no probabilístico para determinar la población, significó la adopción de un criterio subjetivo e implicó: a) un esfuerzo deliberado para obtener una muestra representativa mediante la inclusión de sujetos típicos; y b) la optimización de los recursos disponibles. Sin embargo, no resultó arbitrario, sino de la conjunción de antecedentes relevantes y pertinentes, lo que acotó las limitaciones emergentes de este tipo de muestreo.

2) El muestreo probabilístico utilizado en la extracción de la muestra aceptante, concede mayor rigurosidad metodológica y representatividad. Así, las conclusiones obtenidas explican a toda la población aunque no permitirían inferir sobre el universo.

Unidad de análisis: La definición del pequeño productor sojero chaqueño como unidad de análisis, se debe a su importancia económico-social y su vulnerabilidad.

Fuente de datos: La escasa capacidad para actuar sobre (o transformar) el objeto de investigación, como así también la posibilidad real y valiosa de acceder al productor sojero en forma directa, determinaron una fuente de datos testimonial, proveniente exclusivamente de manifestaciones ${ }^{4}$ del agricultor ante las distintas preguntas y aclaraciones efectuadas, utilizando como técnica la encuesta y como instrumento de observación un cuestionario-entrevista descripto en el punto 2.4 .

\subsection{Variables consideradas en el estudio}

Las variables teóricas se desprenden de la definición de explotación sustentable (protección de los recursos naturales, rentabilidad y aceptación social); que se corresponden, con variables empíricas. La rentabilidad se evidencia a partir del volumen de producción (medición física), los ingresos y gastos (medición financiera); en tanto que la aceptación social se observa a través de la situación legal de la tierra explotada (propiedad, arrendamiento, usurpación, en-

\footnotetext{
${ }^{4}$ En ningún caso se solicito respaldo documental para verificar la información proporcionada.
} 
tre otros) y el empleo de recursos humanos, como hechos indicativos del accionar del pequeño productor. Los resultados obtenidos en el análisis de los datos recolectados se indican en el tercer apartado de este trabajo.

\subsection{Instrumento de medición}

Con el propósito de recoger los datos relativos al tema bajo estudio se diseño ad hoc un instrumento denominado "Cuestionario de Sustentabilidad en Pequeñas Explotaciones Agrícolas Sojeras de la Provincia del Chaco (PEASPC)”, el que pude consultarse en Gusinsky et al. (2013). El mismo consta de cuatro bloques, con las siguientes características: el primero identifica a los productores, el segundo tiene catorce preguntas cerradas que se responden calificando sobre la base de una escala tipo Likert de 5 puntos que incluye las opciones: Desconoce (1), Nunca (2), Pocas veces (3), Muchas veces (4), Permanentemente (5). El tercer grupo de preguntas registra datos numéricos de tres campañas agrícolas y como mínimo se anotan los que corresponden a la última. En el cuarto bloque, se encuentran cinco ítems, que amplían detalles de ciertas decisiones adoptadas. En aquellos casos en los que las respuestas correspondían a datos cualitativos (no métricos); es decir, medidas en escalas de tipo nominal y ordinal, al momento de procesar la información relevada, fue necesario cuantificarlas.

El instrumento de medición ha sido validado cualitativamente mediante los métodos: a) juicio de expertos y b) grado de acuerdo. El primero, proporciona indicadores subjetivos de validez y el segundo aporta lo que algunos autores llaman indicadores de la validez factorial. La evaluación es necesaria y relevante pues indica en que grado la prueba será capaz de medir lo que realmente pretende, cuyas condiciones de aplicación se describen en el próximo apartado.

\subsection{Procedimiento}

La recolección de datos es una instancia delicada en toda investigación empírica, en virtud de ello, se expone seguidamente un resumen del procedimiento aplicado.

En primer lugar, la colaboración de delegados de la Agencia de Desarrollo Rural del Ministerio de Producción del Gobierno de la Provincia, facilitó la ubicación geográ-fica de las explotaciones sojeras.

El trabajo de campo propiamente dicho, se llevó a cabo durante septiembre de 2012, mediante entrevistas cuya duración promedio fue de treinta minutos, realizadas en la residencia de los productores. Esta tarea estuvo a cargo de tres docentes-investigadores, previa consulta con los mismos, a fin de acordar el horario de los encuentros. En el primer contacto, se les informó que su colaboración voluntaria posibilitaría la realización de una investigación desarrollada en el marco de un proyecto acreditado por la Universidad Nacional del Nordeste; y que los datos recogidos serían manejados con carácter estrictamente confidencial y utilizados solamente con fines científicos. Se les indicó también, la importancia de responder sinceramente. 
El relevamiento transcurrió con absoluta normalidad, no habiéndose recibido ningún tipo de sugerencias o expresiones relevantes, por parte de los productores encuestados. Su finalización, dio paso al ordenamiento de la información obtenida y a la construcción de la base de datos en formato electrónico.

\section{3) RESULTADOS}

Una de las bondades que caracteriza a la investigación empírica es que permite contrastar el concepto teórico que se sostiene con los datos de la muestra; es decir, brinda la oportunidad de evaluar, en la realidad, el grado de ajuste entre el paradigma que se hipotetiza y los resultados que se obtienen.

Superado el control de la matriz de datos y, con el propósito de obtener información sobre las particularidades que presenta el tema objeto de interés, se han realizado -con asistencia del programa informático SPSS 20.0- los análisis estadístico-descriptivos que se indican en Tabla 1. Todo ello a fin de explorar el comportamiento de las variables y comprobar en qué medida los resultados alcanzados están en consonancia con los proporcionados por la literatura especializada.

Tabla 1. Análisis estadísticos implementados en esta investigación

\begin{tabular}{|c|l|l|}
\hline Dominio & Estudios estadísticos & Indicadores \\
\hline \multirow{3}{*}{ Descriptivo } & * De tendencia central & Media, moda y mediana. \\
\cline { 2 - 3 } & *De dispersión & Desviación estándar, mínimo, máximo y rango. \\
\cline { 2 - 3 } & *De distribución & Tablas de frecuencias, asimetría y curtosis. \\
\hline
\end{tabular}

Dado el número de variables que se estudian, los análisis estadísticos que podrían tener lugar en este apartado son numerosos. Sin embargo, se presentarán sintéticamente aquellos indicadores que han parecido más adecuados para caracterizar la muestra en las diferentes variables a nivel de centralización, dispersión y distribución.

En virtud de lo expuesto, se incluyen a continuación: a) una reseña de los resultados obtenidos referidos a las variables conceptuales: rentabilidad y aceptación social, que resultaron útiles para describir particularidades de las explotaciones sojeras chaqueñas, véase puntos 3.3. y 3.4. respectivamente y; b) las conclusiones, basadas en un estudio similar implementado oportunamente, sobre datos personales y protección del recur-so natural suelo (Gusinsky et al., 2013).

\subsection{Resultados obtenidos relativos a datos personales}

Los productores agrícolas encuestados poseen una edad promedio de 48.70, con una desviación estándar de 12.91; predominando el sexo masculino (92.59\%) entre ellos. Por otra 
parte, cabe señalar, que sus explotaciones agrícolas se encuentran ubicadas en su mayor parte en el Departamento Chacabuco (51.72\%), cuya ciudad cabecera es Charata.

\subsection{Resultados obtenidos vinculados con la protección del suelo}

Los participantes del estudio demostraron conductas permanentes que propenden a la sustentabilidad, tales como: rotación de cultivos, implantación sobre rastrojo, adopción de siembra directa y semilla transgénica, como también el uso de agroquímicos y el monitoreo de plagas bajo asistencia técnica. Sin embargo, se debe fortalecer la alternancia de actividades económicas (ej. agricultura-ganadería), la incorporación de fertilización balanceada y la realización periódica del estudio de suelo, tendientes al cuidado preventivo del mismo.

\subsection{Resultados obtenidos ligados a la rentabilidad}

Como ya se indicó en la introducción, la rentabilidad se obtiene a partir de la sumatoria algebraica de las causas generadoras de resultado.

Para ponderar mejor esta variable, hubiese sido de mucha utilidad la determinación cuantitativa del punto de equilibrio a partir del cual el cultivo es rentable, sin embargo, dada la reticencia lógica de los entrevistados a brindar datos de tipo patrimonial, el cuestionario carecía de los ítems necesarios para obtener las respuestas pertinentes que permitirían implementar el análisis numérico recomendado.

En su reemplazo se les solicitó a los productores su apreciación general acerca del beneficio obtenido. En este caso, su respuesta pudo estar sesgada por la confusión habitual entre excedente financiero y rentabilidad; siendo ésta última abarcativa de todos los componentes que determinan el resultado económico de la empresa, inclusive los que no tienen correlato en el flujo de fondos del período (ej. depreciaciones).

Las respuestas se indican en Tabla 2. Resulta notorio que el 51.86\% de los entrevistados ha logrado un resultado satisfactorio pocas veces, lo que sumado al 7.40\% de los individuos que consideran nunca haber tenido beneficios con la explotación de soja, conforman el 59.26\% de la muestra. Estas experiencias, indudablemente, contrastan con el imaginario colectivo sobre la explotación del cultivo.

Tabla 2: Beneficio satisfactorio de la explotación de soja.

\begin{tabular}{|l|l|l|l|}
\hline Categorías & Frecuencia & Porcentaje & Porcentaje acumulado \\
\hline Nunca & 2 & 7.40 & 7.40 \\
\hline Pocas veces & 14 & 51.86 & 59.26 \\
\hline Muchas veces & 7 & 25.92 & 85.18 \\
\hline Permanentemente & 4 & 14.82 & 100.00 \\
\hline Total & 27 & 100.00 & \\
\hline
\end{tabular}


La cadena de comercialización de soja se extiende desde el pequeño productor (sin condiciones para exportar) a los acopiadores y luego a los agroexportadores.

La principal empresa exportadora de grano de soja, harina y aceite es: Cargill, (Giancola, Salvador, Covacevich e Iturrioz, 2009), en menor importancia relativa comparten la tabla Bunge Argentina S.A., Aceitera General Deheza (AGD) y Dreyfus entre otras. La comercialización directa a estos agentes productivos o el envío al Puerto de Rosario para exportación, evidencia la búsqueda de mejores condiciones de venta, avanzando hacia otros actores de la cadena de valor. Sin embargo, puede apreciarse en Tabla 3 que sólo un productor de la muestra se identifica con esta alternativa.

Tabla 3: Venta de producción sojera a acopiadores locales.

\begin{tabular}{|l|l|l|l|}
\hline Categorías & Frecuencia & Porcentaje & Porcentaje acumulado \\
\hline Muchas veces & 1 & 3.70 & 3.70 \\
\hline Permanentemente & 26 & 96.30 & 100.00 \\
\hline Total & 27 & 100.00 & \\
\hline
\end{tabular}

Una característica distintiva de la actividad agrícola es su fuerte exposición a contingencias de tipo climático que determinan el resultado final de la explotación. En estos últimos años se ha avanzado en el diseño, difusión y comercialización de seguros que resguardan la producción ante la ocurrencia de los eventos incluidos en la póliza. En Tabla 4 se muestran las observaciones realizadas respecto de este ítem.

Tabla 4: Contratación seguro agrícola

\begin{tabular}{|l|l|l|l|}
\hline Categorías & Frecuencia & Porcentaje & Porcentaje acumulado \\
\hline Nunca & 12 & 44.45 & 44.45 \\
\hline Pocas veces & 7 & 25.92 & 70.37 \\
\hline Muchas veces & 0 & 0.00 & 70.37 \\
\hline Permanentemente & 8 & 29.63 & 100.00 \\
\hline Total & 27 & 100.00 & \\
\hline
\end{tabular}

Los hallazgos indican que el 44.45\% de los pequeños productores, nunca contrató seguro agrícola, sin poder discriminar si dicha decisión está vinculada a una falta de conocimiento respecto del mercado asegurador ó al costo del producto. Respecto del 55.55\% restante, es evidente que conoce las coberturas ofrecidas, toda vez que han contratado, al menos una vez, el seguro agrícola; y el 29.63\% toma esta decisión permanentemente, adoptando una alternativa de gestión que contribuye a la disminución del riesgo típico de la actividad y la estabilización de los resultados en el tiempo. Estos efectos se producen porque el seguro proporciona un resguardo ante la naturaleza incontrolable del clima, minimizando el impacto patrimonial, 
económico y financiero que provoca la ocurrencia del evento asegurado.

El estudio de rentabilidad se profundiza con la recolección de datos respecto del volumen de producción y del precio obtenido en la últimas tres campañas (a septiembre/2012), bajo el supuesto que el productor realiza una sola plantación anual de soja (p. ej., la campaña 2011/2012, se correspondió con la plantación realizada en noviembre-diciembre de 2011 y cosechada en abril-mayo de 2012).

Asimismo cabe aclarar que, en este trabajo, se sometieron a procesamiento las observaciones relativas a las campañas 2010/2011 y 2011/2012, como referentes de una buena y mala campaña respectivamente. Respecto a la del 2009/2010, si bien han sido relevados algunos datos, los mismos fueron desestimados dado que: a) presentaban características muy similares, en sus diferentes ítems, a las del año 2011 con lo cual en el análisis de las diferencias entre un año y el otro eran poco relevantes; b) varios productores encuestados no recordaban con precisión la información que era requerida, dada la antigüedad de tales datos.

Para ilustrar lo mencionado precedentemente, como también lo que ha sucedido con la producción de soja en los últimos cinco años en la Provincia del Chaco, se incluyen en Tabla 5 los datos globales para cada período.

Tabla 5: Superficie y producción de soja en la Provincia del Chaco

\begin{tabular}{|l|l|l|l|l|l|}
\hline Campañas & $2009 / 2010$ & $2010 / 2011$ & $2011 / 2012$ & $2012 / 2013$ & $2013 / 2014$ \\
\hline Superficie sembrada (ha) & 668.600 & 700.800 & 689.550 & 548.230 & 591.300 \\
\hline Superficie cosechada (ha) & 642.450 & 698.520 & 386.150 & 423.580 & 576.330 \\
\hline Producción total (tn) & 1.550 .860 & 1.655 .117 & 294.589 & 553.794 & 1.384 .537 \\
\hline Rendimiento promedio (kg/ha & 2.414 & 2.369 & 763 & 1.307 & 2.402 \\
\hline
\end{tabular}
Fuente: Elaboración propia con datos del Ministerio de Agricultura, Ganadería y Pesca de la Repú-
blica Argentina (s/f)

En Tabla 6 se muestran los resultados de los indicadores de centralización, dispersión y distribución relacionados con el volumen de producción informado por los participantes del estudio. Éstos se complementan con Tablas 7 y 8 (frecuencias).

Tabla 6: Volumen de producción.

\begin{tabular}{|l|r|r|}
\hline Indicadores calculados & $\begin{array}{c}2010 / 2011 \\
n=27\end{array}$ & $\begin{array}{c}2011 / 2012 \\
n=27\end{array}$ \\
\hline Media & 2031.48 & 344.07 \\
\hline Moda & 2000.00 & 0.00 \\
\hline Desviación estándar & 813.25 & 344.38 \\
\hline Asimetría & -0.74 & 0.90 \\
\hline Curtosis & 1.78 & 0.25 \\
\hline Rango & 3500.00 & 1200.00 \\
\hline Mínimo & 0.00 & 0.00 \\
\hline Máximo & 3500.00 & 1200.00 \\
\hline
\end{tabular}

Nota: Los volúmenes de producción están expresados en kg/ha. 
Como puede apreciarse, según datos estadísticos del Ministerio de Agricultura, Ganadería y Pesca de la República Argentina, para la campaña 2010/2011 el rendimiento promedio ha sido de 2369 kilogramos por hectárea cosechada (véase Tabla 5); este valor es levemente superior al determinado para la muestra $(2031.48 \mathrm{~kg} / \mathrm{ha})$. En ese período, el rango de producción fue de $3500 \mathrm{~kg}$, en tanto que el rendimiento modal (de mayor frecuencia) fue de 2000 kilogramos por hectárea. A su vez, el estadístico de la curtosis (Curt $=1.78)$ indica que el grado de concentración que presentan los valores en la región central de la distribución es muy elevada, marcando la paridad de los rendimientos en el $51.86 \%$ de la muestra. Por todo lo indicado precedentemente, la producción normal de pequeños productores sojeros chaqueños ha sido de $2000 \mathrm{~kg} / \mathrm{ha}$.

Tabla 7: Volumen de producción 2010/2011

\begin{tabular}{|l|c|c|c|}
\hline \multicolumn{1}{|c|}{$\mathrm{kg} / \mathrm{ha}$} & Frecuencia & Porcentaje & Porc. acumul. \\
\hline $0.00-1000$ & 3 & 11.11 & 11.11 \\
\hline $1000-2000$ & 14 & 51.86 & 62.97 \\
\hline $2000-3000$ & 8 & 29.63 & 92.60 \\
\hline $3000-4000$ & 2 & 7.40 & 100.00 \\
\hline Total & 27 & 100.00 & \\
\hline
\end{tabular}

Fuente: Elaboración propia.
Tabla 8: Volumen de producción 2011/2012

\begin{tabular}{|c|c|c|c|}
\hline $\mathrm{kg} / \mathrm{ha}$ & Frecuencia & Porcentaje & Porc. acumul. \\
\hline $0.00-300$ & 15 & 55.56 & 55.56 \\
\hline $300-600$ & 9 & 33.33 & 88.89 \\
\hline $600-900$ & 0 & 0.00 & 88.89 \\
\hline $900-1200$ & 3 & 11.11 & 100.00 \\
\hline Total & 27 & 100.00 & \\
\hline
\end{tabular}
Fuente: Elaboración propia.

En la campaña 2012, la moda resultó 0.00 , toda vez que el mayor estrato de producción obtenida se ubica entre cero y trescientos $\mathrm{kg} / \mathrm{ha}$ (55.56\%), es decir, un rendimiento nulo (o ínfimo), que se corresponde con situaciones donde se perdió toda la producción debido a la fuerte sequía (tanto por el evento climático mismo como por el hecho de ser antieconómica la recolección de la magra producción restante) o, en el mejor de los casos, no se plantó soja en el período observado. Esto es consistente con el coeficiente de curtosis (Curt $=0.25)$ que indica una distribución muy similar a una curva normal, ya que esta dentro del rango de tolerancia aconsejado $[-0.5,0.5]$. A su vez, complementa su interpretación, una prolongada inclinación de la curva hacia la derecha $($ As $=0.90)$ dada por la dispersión de la muestra restante. Los valores de rendimiento promedio provincial, para este período, han duplicado la media de la muestra, pero más allá del valor absoluto, es indudable que la producción tuvo un fuerte golpe del cual le ha costado dos años recuperarse (véase Tabla 5).

Las respuestas de aquellos productores, dispuestos a informar el precio de venta obtenido, permitieron la determinación de los indicadores que se detallan en Tabla 9. 
Tabla 9: Precio de venta obtenido.

\begin{tabular}{|l|r|r|}
\hline \multirow{2}{*}{ Indicadores } & \multicolumn{2}{|c|}{ Productores $(\mathrm{n}=27)$} \\
\cline { 2 - 3 } & $\begin{array}{c}2011 \\
n_{1}=19\end{array}$ & $\begin{array}{c}1 \\
2012 \\
n_{2}=23\end{array}$ \\
\hline Media & 781.08 & 930.52 \\
\hline Moda & 0.00 & $900.00(\mathrm{a})$ \\
\hline Desviación estándar & 654.93 & 379.23 \\
\hline Asimetría & -0.34 & -1.64 \\
\hline Curtosis & -1.90 & 2.34 \\
\hline Rango & 1500.00 & 1300.00 \\
\hline Mínimo & 0.00 & 0.00 \\
\hline Máximo & 1500.00 & 1300.00 \\
\hline
\end{tabular}

(a) Existen múltiples modas, se seleccionó la que mejor describe la realidad objeto de interés.

Fuente: Elaboración propia.

Nota: Los precios están expresados en pesos por tonelada.

Los datos recolectados sobre el precio en 2011, arrojaron un índice de curtosis que ha expuesto el elevado grado de concentración de los valores en la región central de la distribución $($ Curt $=2.34$, superior al nivel de tolerancia de o.5), asimismo como la asimetría es negativa $(-1.64)$ la curva está sesgada a la izquierda. Esto permite inferir un precio de venta similar para una gran parte de los productores encuestados, cuyo valor, teniendo en cuenta la moda, oscilaría alrededor de los novecientos pesos por tonelada. Completa esta apreciación, la interpretación combinada de la media (\$930.52), la desviación estándar (\$ 379.23) y el precio máximo (\$1300.0o). Los valores de tales indicadores permiten asumir que -como mínimoel 68.26\% de los entrevistados obtuvo un precio ubicado entre $\$ 1300.00$ y $\$ 551.29$.

En 2012, los valores de la variable bajo estudio se presentan de manera muy dispersa en la región central de la distribución (Curt $=-1.90)$. Esto se debe a que la mayor parte de los empresarios no pudo vender su producción (sequía), y los pocos que levantaron alguna cosecha obtuvieron una media de $\$ 781.08$, con poca concentración del precio $(D E=654.93)$.

Por otra parte, los precios informados por la Cámara Arbitral de Cereales de Rosario, desde el o1 de abril al 31 de agosto de 2011, crecieron desde un mil doscientos ochenta y ocho (\$1288.00) a un mil trescientos noventa ( $\$ 1390.00)$ pesos por tonelada de soja, respectivamente. Para idéntico período del 2012 los precios publicados han aumentado desde un mil quinientos (\$1500.00) a dos mil (\$2000.00) pesos por tonelada de soja.

La comparación de los precios entre el mercado de Rosario y el valor obtenido por el productor chaqueño ha permitido observar que en una campaña agrícola:

1) Buena (2010/2011), los agricultores recibieron en su mayor parte, un 70.00\% ( $\$$ 900.00/ \$ 1288.00) del valor de cotización del producto en el mercado.

2) Mala (2011/2012), los pocos productores que cosecharon obtuvieron como máximo el $52 \%$ (\$ 781/\$ 1500) del valor de mercado. 
La diferencia de precios, muy significativa en 2012, ha puesto en evidencia otros elementos que, sin duda, han incidido en la rentabilidad de la explotación sojera tales como el costo de transporte e intermediación y la calidad del grano cosechado.

\subsection{Resultados obtenidos relacionados con la aceptación social}

En este apartado se analizan las respuestas a preguntas del cuestionario de sustentabilidad en PEASPC que permiten observar la relación de la explotación agrícola con su comunidad.

A los efectos de este estudio, se han considerado como aspectos sociales positivos: ser propietario de la tierra, vivir dignamente de la explotación agrícola (productor y su familia) y contratar personal, con preferencia en forma permanente.

Las preguntas han previsto también, la obtención de evidencias confirmatorias sobre las restricciones impuestas a los participantes del estudio, a saber: a) la explotación no supera 500 ha de superficie, 200 ha de superficie cultivada y 2 empleados no familiares permanentes; b) los productores son personas físicas (empresas unipersonales).

Los resultados del análisis de los datos recabados para las variables que dan cuenta de la aceptación social de la explotación sojera, se muestran, en Tablas 10 a 12.

Tabla 10: Recurso tierra.

\begin{tabular}{|l|c|c|c|c|c|c|}
\hline \multirow{2}{*}{$\begin{array}{c}\text { Indicadores } \\
n=27\end{array}$} & \multicolumn{2}{|c|}{$\begin{array}{c}\text { Hectáreas } \\
\text { disponibles }\end{array}$} & \multicolumn{2}{c|}{$\begin{array}{c}\text { Hectáreas destinadas } \\
\text { a la agricultura }\end{array}$} & $\begin{array}{c}\text { Hectáreas propias destinadas } \\
\text { a la agricultura }\end{array}$ \\
\cline { 2 - 7 } & 2011 & 2012 & 2011 & 2012 & 2011 & 2012 \\
\hline Media & 128.77 & 124.92 & 125.77 & 123.85 & 80.96 & 77.11 \\
\hline Moda & $120.00(\mathrm{a})$ & $120.00(\mathrm{a})$ & $120.00(\mathrm{a})$ & $120.00(\mathrm{a})$ & 0.00 & 0.00 \\
\hline Desv. estándar & 46.37 & 49.33 & 43.84 & 48.02 & 60.80 & 62.61 \\
\hline Rango & 160.00 & 200.00 & 160.00 & 200.00 & 200.00 & 200.00 \\
\hline Mínimo & 40.00 & 0.00 & 40.00 & 0.00 & 0.00 & 0.00 \\
\hline Máximo & 200.00 & 200.00 & 200.00 & 200.00 & 200.00 & 200.00 \\
\hline
\end{tabular}

(a)Existen múltiples modas, se indica la menor de ellas.

Fuente: Elaboración propia.

De la observación del valor modal de las respuestas a cada pregunta formulada, (Tabla 10) resulta llamativo que: el mayor número de productores encuestados dispuso de 120 hectáreas, las que destinó a la agricultura, pero no eran de su propiedad. Estos indicadores, han evidenciado que los dueños de la tierra han abandonado el oficio de agricultores, prefiriendo arrendarlas como modo alternativo de sustento.

En efecto, según la literatura específica (Fundapaz, 2007), hasta el momento -en cuanto a producción en los departamentos sojeros chaqueños- los propietarios disminuyeron un $30 \%$, mientras que los arrendamientos y otros contratos aumentaron un $50 \%$, lo que refleja la nueva organización de la producción, así como el ingreso de nuevos actores (arrendatarios).

Por otra parte, según datos del Censo Nacional 2002, las explotaciones agropecuarias en el 
Chaco han sido 16.898, de las cuales el 87\% (14.730) fueron familiares con una superficie media de 162 hectáreas (Scheinkerman, 2009), valor levente superior a la media surgida de este estudio.

Con relación a los integrantes de la familia que viven y/o trabajan en la explotación, se han calculado los indicadores que se resumen en Tabla 11.

Tabla 11: Miembros de la familia que viven y/o trabajan en la explotación

\begin{tabular}{|l|c|c|c|c|}
\hline \multirow{2}{*}{$\begin{array}{c}\text { Indicadores } \\
n=27\end{array}$} & \multicolumn{2}{|c|}{ Viven en la explotación } & \multicolumn{2}{c|}{ Trabajan en la explotación } \\
\cline { 2 - 5 } & 2011 & 2012 & 2011 & 2012 \\
\hline Media & 1.63 & 1.67 & 1.37 & 1.41 \\
\hline Moda & 0.00 & 0.00 & 1.00 & 1.00 \\
\hline Desv. estándar & 1.67 & 1.64 & 1.14 & 1.11 \\
\hline Rango & 5.00 & 5.00 & 4.00 & 4.00 \\
\hline Mínimo & 0.00 & 0.00 & 0.00 & 0.00 \\
\hline Máximo & 5.00 & 5.00 & 4.00 & 4.00 \\
\hline
\end{tabular}

Fuente: Elaboración propia.

En Tabla 11, el valor modal cero indicó que ninguno de los integrantes de la familia vive en el establecimiento, y que sólo uno de ellos (el productor) trabaja en él. Este resultado ha demostrado el éxodo que se produjo desde el campo hacia distintos centros poblados como Charata, General Pinedo, Las Breñas, entre otros. La razón de este desplazamiento ha sido, quizás, la búsqueda de una mejor calidad de vida (particularmente educación), y ha generado un fenómeno que impide a las nuevas generaciones desarrollar un sentido de pertenencia y adquirir el saber hacer de la actividad agrícola.

Con respecto al número de personal contratado, en atención a que no hubo modificaciones en los períodos bajo análisis (2010/2011 y 2011/2012), se ha presentado la información inherente a cada concepto de manera unificada. Asimismo, las respuestas permitieron la construcción del indicador de recursos humanos necesarios para una explotación sojera; todos los valores pueden observarse en la Tabla 12.

Tabla 12: Recursos humanos afectados a la explotación de soja

\begin{tabular}{|l|c|c|c|}
\hline $\begin{array}{c}\text { Indicadores } \\
n=27\end{array}$ & $\begin{array}{c}\text { Personal } \\
\text { permanente }\end{array}$ & $\begin{array}{c}\text { Personal } \\
\text { temporario }\end{array}$ & $\begin{array}{c}\text { Recursos } \\
\text { humanos }\end{array}$ \\
\hline Muestra & 27 & 27 & 27 \\
\hline Media & 0.11 & 0.44 & 1.96 \\
\hline Moda & 0.00 & 0.00 & 2.00 \\
\hline Desviación estándar & 0.32 & 0.51 & 1.16 \\
\hline Rango & 1.00 & 1.00 & 5.00 \\
\hline Mínimo & 0.00 & 0.00 & 0.00 \\
\hline Máximo & 1.00 & 1.00 & 5.00 \\
\hline
\end{tabular}

Fuente: Elaboración propia. 
La facilidad de las tareas culturales involucradas en el proceso (siembra y cosecha), como también la tecnología avanzada requerida para llevar adelante este cultivo en condiciones sustentables, hace que se empleen de manera directa -en promedio- sólo una persona por cada cien hectáreas trabajadas.

Esto puede apreciarse en el indicador de recursos humanos, construido a partir de la suma de la cantidad de personas (miembros de la familia y personal contratado) que trabajan para llevar adelante la explotación sojera. Si bien el rango de personas que se emplean productivamente varía entre cero y cinco, la media y la moda de la muestra se ubican en dos personas. Como los productores explotan hasta un máximo de doscientas hectáreas, la relación es ciertamente una persona cada cien hectáreas cultivadas.

\section{4) DISCUSIÓN}

La discusión de resultados se realiza con el propósito de que sea el punto de partida para presentar, a través de las conclusiones, los aportes más relevantes que este estudio anhela realizar a la comunidad científica y profesional. En ese sentido, se presentan: a) algunas apreciaciones acerca de las estimaciones realizadas, b) el planteo de investigaciones complementarias, y c) las propuestas de intervención.

\subsection{Apreciaciones finales acerca de las estimaciones realizadas}

Debido a los numerosos análisis estadísticos realizados para estudiar adecuadamente las variables del cuestionario ad hoc, se ha generado una cantidad considerable de información, por lo que no fue una tarea sencilla resumirla y reflexionar con ecuanimidad respecto de los resultados producidos sobre la rentabilidad y aceptación social de una empresa sojera chaqueña.

Los mismos han sido conseguidos a partir de las medidas recogidas, en atención a ello no serían generalizables puesto que responden a las características particulares de la muestra seleccionada para la presente investigación.

Así pues, del análisis de datos surge que, en 2010/2011 en Chaco, se obtuvo un rendimiento promedio de 2369 kilogramos de soja por hectárea cosechada; mientras que los productores de la muestra obtuvieron una media de $2031.48 \mathrm{~kg} / \mathrm{ha}$. Esta situación, no puede ser generalizable; sin embargo, podría inferirse que: en gran número las pequeñas explotaciones sojeras obtienen rendimientos menores a la media provincial.

El desarrollo que ha tenido éste trabajo en la etapa empírica -en lo atinente a rentabilidad y aceptación social-, permite señalar que ha sido posible:

1) Explorar el comportamiento de las variables del cuestionario aplicado, a través del tratamiento de los datos obtenidos, lo que derivó en un gran número de indicadores descrip- 
tivos que resultan de utilidad para conocer, mediante información fehaciente, adecuada y actual, la caracterización que presentan las pequeñas empresas radicadas en el polo sojero del Oeste del Chaco, como provincia que no pertenece a la región pampeana.

2) Comprobar que los resultados alcanzados se aproximan a los proporcionados por la literatura específica en la temática bajo análisis, explicando empíricamente una serie de presunciones teóricas que, implícita o explícitamente, se hallaban en cada una de los ítems evaluados. Sin embargo, dicha contrastación cuantitativa, fue posible sólo en los casos en que se encontró información confiable para establecer la comparación.

\subsection{Planteos de investigaciones complementarias}

Con el objetivo de generar pautas objetivas para la evaluación de la sustentabilidad económica y social en explotaciones sojeras, se considera conveniente efectuar los siguientes planteos de investigaciones complementarias:

1) Ampliar el estudio a zonas de similares características sociales, económicas y culturales, para comparar si se repiten (o no) los patrones de comportamiento observados.

2) Proyectar la metodología a nuevos períodos de observación (diseño de tipo longitudinal), y de corresponder, la adecuación del instrumento de medición.

3) Replicar la captura de datos en otros cultivos, dado que muchas de las cuestiones señaladas y tratadas a lo largo de este trabajo son comunes.

4) Planear la elaboración del "Indicador de Sustentabilidad (IS)" que califique periódicamente a las empresas agrícolas. Se obtendría mediante la relación de las variables estudiadas, debidamente tabuladas y ponderadas según la importancia de su incidencia en modelo de sustentabilidad.

\subsection{Propuestas de intervención}

A partir de los resultados obtenidos, se brindan a continuación propuestas de intervención, que se pretende, contribuyan con el desarrollo de las pequeñas empresas sojeras del Chaco.

1) Concientizar a los actores del sector acerca de la importancia que tiene el desarrollo de una explotación económica y social sustentable.

2) Fomentar la construcción de bases de datos, por parte de organismos e instituciones tanto públicas como privadas, que permitan disponer de información a efectos de la realización de estudios sobre la materia objeto de interés.

A fin de fortalecer la comunidad agrícola, sería conveniente la puesta en marcha de estas medidas de acción, absolutamente plausibles de implementar. 


\section{5) CONCLUSIONES}

En Chaco, se ha fortalecido permanentemente el polo sojero situado en el sudoeste de la provincia, con epicentro en las localidades de Charata y General Pinedo separadas a una distancia de quince kilómetros aproximadamente.

La investigación realizada tuvo como objetivo general evaluar la sustentabilidad de las empresas sojeras Chaqueñas. Bajo esa premisa, el instrumento fue diseñado para la observación sistemática de las tres variables teóricas consideradas en la definición de explotación sustentable y ha proporcionado datos muestrales de cada una de ellas. Por cuestiones fácticas, la publicación de los resultados ha debido hacerse en dos etapas, situación que explica la gran similitud de ambos artículos en lo que a diseño estructural y metodológico se refiere. Este estudio se concentra en los resultados obtenidos a partir del análisis de datos efectuado para las variables: rentabilidad y aceptación social; encontrándose publicados (Gusinsky et al., 2013), los atinentes a la protección del recurso natural suelo. Asimismo, han permitido la consecución del objetivo, como paso necesario para validar la hipótesis sustantiva oportunamente formulada.

Cabe resaltar que la totalidad de las variables que intervinieron en el cuestionario elaborado resultaron útiles a fin de brindar una reseña sobre las particularidades de las explotaciones sojeras chaqueñas. A continuación se explicita la información descriptiva basada en el análisis cuantitativo implementado en el presente trabajo.

Para la variable rentabilidad, los resultados indican que gran parte de los productores (59.26\%) se manifiesta disconforme con el resultado económico obtenido. Un porcentaje más bajo que el anterior (44.45\%) desiste de la contratación de coberturas de riesgo y la casi totalidad de ellos (96.30\%) comercializa su producción por medio de acopiadores locales. A su vez, los datos capturados sobre el volumen de producción y precio, han explicado las siguientes situaciones:

1) Período 2010/2011 (referente de una buena campaña): el rendimiento promedio provincial $(2369 \mathrm{~kg} / \mathrm{ha})$ fue levemente superior al determinado para la muestra (2031.48 kg/ha) y el máximo volumen de producción trepó a $3500 \mathrm{~kg} /$ ha. La elevada concentración de los valores en la región central de la distribución ha marcado la paridad de los rendimientos en gran parte de la muestra. Así, el valor de mayor recurrencia fue $2000 \mathrm{~kg} / \mathrm{ha}$, el cual puede considerarse representativo de la producción normal del pequeño productor sojero chaqueño. El precio ha oscilado en torno a los novecientos pesos por tonelada, lo que representó un 70.00\% del valor del producto en el mercado de cereales de Rosario.

2) En 2011/2012 (referente de una mala campaña): el rendimiento promedio provincial marcó un brusco descenso como consecuencia de la fuerte sequía registrada en ese período. Aún así ha duplicado la media de la muestra, pero más allá del valor absoluto, es indudable que la producción de esta oleaginosa tuvo un fuerte golpe 
recuperándose a valores normales recién en la campaña 2013/2014. Los pocos productores que levantaron alguna cosecha, obtuvieron un promedio de 781.08 \$/tn, con gran dispersión del precio $(D E=654.93)$, lo que a su vez, representó aproximadamente el $52.00 \%$ del valor del producto en el mercado de cereales de Rosario. 3) La diferencia de precios, entre lo que recibe el productor y el valor del producto en el mercado de cereales de Rosario, se debe a conceptos que, sin duda, inciden en la rentabilidad de la explotación sojera tales como costos de transporte e intermediación y la calidad del grano cosechado.

Para la variable aceptación social, el estudio de los valores de mayor recurrencia (Moda) ha indicado que los pequeños productores disponen de 120 hectáreas no propias que destinan a la agricultura. Su familia no vive ni trabaja en el campo, y sus integrantes fueron traccionados hacia las ciudades; fenómeno que impide a las nuevas generaciones desarrollar un sentido de pertenencia y adquirir el saber hacer de la actividad agrícola. La cantidad de personas ocupadas en explotaciones sojeras de hasta doscientas hectáreas oscila entre cero y cinco, siendo dos el valor más frecuente, lo que da lugar a establecer una relación directa de: una persona cada cien hectáreas cultivadas.

Finalmente, más allá de las limitaciones de recursos materiales y humanos que todo trabajo de investigación conlleva, se espera que este primer acercamiento, por nuestra parte, a la temática tratada constituya un aporte a la explotación sojera sustentable. Desde luego, también se anhela que este estudio represente una contribución razonable para la comunidad científica y profesional abocada a su asesoramiento e interesada en llevar a cabo futuras investigaciones en esta área disciplinar.

\section{REFERENCIAS BIBLIOGRÁFICAS}

Casparri, M.T. y García Fronti, V. (2011). Los pequeños productores y los seguros agrícolas: encuesta exploratoria. En M. T. Casparri y A. S. Vilker (Eds.). Finanzas Agropecuarias en un contexto de incertidumbre (pp. 57-68). Buenos Aires, Argentina: Universidad de Buenos Aires.

Gusinsky, S. P., Cruz, R. T., Carbajal, M. C., Jerez, S. R. y de Castro, I. G. (2013). Nivel de sustentabilidad en pequeñas explotaciones sojeras de la provincia del Chaco -suelo agrícola-. Revista de la Facultad de Ciencias Económicas, $\mathrm{N}^{\circ}{ }_{11}$, 83-106. Facultad de Ciencias Económicas de la UNNE, eco.unne.edu.ar/revista/publicaciones/revista_11_AyP_digital.pdf

Giancola, S., Salvador, M., Covacevich, M., e Iturrioz, G. (2009). Análisis de la cadena 
SUSANA PAULINA GUSINSKY DE GELMAN - ANTONIO HUMBERTO CLOSAS - ROSA TERESA CRUZ DE INNOCENTE -

MARGARITA CARLOTA CARBAJAL - SUSANA ROSA JEREZ - IDALIA GABRIELA DE CASTRO

NIVEL DE SUSTENTABILIDAD EN PEQUEÑAS EXPLOTACIONES SOJERAS DEL CHACO (ARGENTINA)

Pags. $102-124$

de soja en la Argentina. Serie estudios e investigaciones $\mathrm{N}^{0}$ 3. Buenos Aires, Argentina: Instituto Nacional de Tecnología Agropecuaria (INTA). Recuperado el 13 de marzo de 2013 de http://inta.gob.ar/documentos/analisis-de-la-cadena-de-soja-en-argentina

Fox, D. (1981). El proceso de investigación en educación. Pamplona, España: EUNSA.

Fundapaz. (2007). Impacto social de la expansión de la frontera agrícola en el Chaco Argentino. Boletín: Puente $N^{o} 66$. Recuperado el 17 de julio de 2012.

Inciarte, R. (2004). Las buenas prácticas agrícolas. Organización de las Naciones Unidas para la agricultura y la alimentación. Oficina Regional de la FAO para América Latina y el Caribe. Recuperado el 14 de noviembre de 2012 de ftp://ftp.fao.org/docrep/fao/o10/AIo10s/AIo10soo.pdf

Ministerio de Agricultura Ganadería y Pesca. Red de Información Agropecuaria Nacional (RIAN). Instituto Nacional de Tecnología Agropecuaria (INTA). (2010). Zonificación RIAN Chaco y Formosa. Recuperado el 14 de diciembre de 2012 de inta.gob.ar/documentos/ zonificacion-rian-de-chaco-formosa/

Ministerio de Agricultura Ganadería y Pesca. Red de Información Agropecuaria Nacional (RIAN). Instituto Nacional de Tecnología Agropecuaria (INTA). (2011, mayo). Informe agropecuario para Chaco y Formosa, por Departamento. Recuperado el 17 de julio de 2012 de inta.gob.ar/documentos/rian-chaco-formosa-informe-mayo-2011/

Ministerio de Agricultura Ganadería y Pesca. (s/f). Información en línea sobre siembra, cosecha, producción y rendimiento de los cultivos. Recuperado el 18 de diciembre de 2014 de: www.siia.gov.ar/_apps/siia/estimaciones/estima2.php

Scheinkerman, E., Foti, M. P.y Román, M. E. (2007). Los pequeños productores de la República Argentina. Importancia en la producción agropecuaria y en el empleo en base al Censo Nacional Agropecuario 2002 (2a. Ed.). Serie estudios e investigaciones $\mathrm{N}^{\circ}$ 10. Buenos Aires, Argentina: Instituto Interamericano de Cooperación para la Agricultura y Ministerio de Agricultura, Ganadería y Pesca de la República Argentina.

Scheinkerman, E. (2009). Las explotaciones agropecuarias familiares de la República Argentina. Un análisis a partir de los datos del Censo Nacional 2002. Serie estudios e investigaciones $\mathrm{N}^{0}$ 23. Buenos Aires, Argentina: Instituto Interamericano de Cooperación para la Agricultura y Ministerio de Agricultura, Ganadería y Pesca de la República Argentina. 


\section{CURRICULUM VITAE}

\section{Susana Paulina Gusinsky de Gelman}

Contadora Pública Nacional (1964), Universidad Nacional de Córdoba. Licenciada en Ciencias Económicas (1967) Universidad Nacional de Córdoba. Profesor Titular de la cátedra: Macroeconomía I (1973 y continúa a la fecha. Dedicación Exclusiva); Facultad de Ciencias Económicas UNNE. Docente de grado y posgrado en temáticas del área Económica; Directora de tesinas de alumnos de grado. Directora y Co directora de proyectos de investigación (2003 a la fecha). Miembro de comisiones asesoras, de evaluación y de concurso. Secretaria de Investigación, Innovación y Desarrollo (2010-2014). Investigador del Programa de Incentivos, categoría II (1999 a la fecha). sgelman@eco.unne.edu.ar

\section{Antonio Humberto Closas}

Se desempeña como Profesor Titular de Estadística II y Director de Proyectos de Investigación en la Facultad de Ciencias Económicas de la UNNE, esta última función también la ejerce en la Facultad Regional Resistencia de la UTN. Ha obtenido el grado de Doctor, mención “cum laude”, por la Universidad Pública de Navarra (Pamplona, España), en el área de Estadística e Investigación Operativa. Es coautor de libros y de diversas publicaciones en revistas de impacto científico nacionales y extranjeras. Es investigador categorizado del Programa de Incentivos, de la Secretaría de Políticas Universitarias, del Ministerio de Educación de la Nación. Participa como conferencista y expositor en reuniones académicas, tanto en Argentina, como en el exterior. Asimismo, es integrante de distintos comités de arbitraje de revistas científicas; también actúa como director o miembro de comisiones de seguimiento y evaluación de tesis de maestrías y doctorados.

hclosas@eco.unne.edu.ar / hclosas@hotmail.com

\section{Rosa Teresa Cruz de Innocente}

Contadora Pública (1978), Profesor en Ciencias Económicas (1987) y Especialista en Sindicatura Concursal (1998); Facultad de Ciencias Económicas de la Universidad Nacional del Nordeste. Profesor Titular de la cátedra: Contabilidad Básica y Profesor adjunto de la cátedra: Estados Contables; Facultad de Ciencias Económicas - UNNE. Docente de grado y posgrado en temáticas del área Contable; Co directora de proyectos de investigación. Miembro de comisiones asesoras, de evaluación y de concurso. Secretaria de Asuntos Estudiantiles y Ex Secretaria de Extensión y Ejercicio Profesional de la Facultad de Ciencias Económicas de la UNNE. Investigador del Programa de Incentivos, categoría IV.

rcruz@eco.unne.edu.ar

\section{Margarita Carlota Carbajal}

Contador Público Nacional (1971); Especialista en Tributación (2000); Profesora en Ciencias Económicas (2004). Facultad de Ciencias Económicas del la Universidad Nacional del Nordeste. 
Máster en Derecho Tributario, Universitat de Barcelona (España), 2002. Profesor adjunto a cargo de la cátedra: Régimen Tributario; Profesor adjunto de la cátedra: Impuestos II. Ex miembro del Consejo Directivo de la Facultad de Ciencias Económicas - UNNE, por el claustro de profesores adjuntos. Jefe Titular de la División Capacitación de la Administración Federal de Ingresos Públicos. Dirección General Impositiva. Región Resistencia (1999 a la fecha) margacarbajal@hotmail.com

\section{Susana Rosa Jerez}

Contadora Pública (1989), Especialista en Contabilidad Superior y Auditoria (2007); Facultad de Ciencias Económicas de la Universidad Nacional del Nordeste. Profesor titular de la cátedra: Contabilidad para Administradores de la Facultad de Ciencias Económicas de la Universidad Nacional del Nordeste y auxiliar docente de la cátedra Estados Contables. Miembro del Consejo Directivo de la Facultad de Ciencias Económicas - UNNE, por el claustro de profesores adjuntos. Integrante de equipos de investigación de proyectos acreditados por la SGCyT de la UNNE, que se llevan a cabo en el ámbito de la Facultad de Ciencias Económicas de la UNNE. Fiscal Relator del Tribunal de Cuentas de la Provincia del Chaco.

\section{su_jerez@hotmail.com / sjerez@eco.unne.edu.ar}

\section{Idalia Gabriela de Castro}

Especialista en Contabilidad Superior y Auditoria, título otorgado por la UNNE en Octubre 2007. Se desempeña como Profesora Adjunta, con dedicación exclusiva, en la cátedra Contabilidad Básica de la Facultad de Ciencias Económicas de la UNNE. Es integrante de equipos de investigación de proyectos acreditados por la Secretaría General de Ciencia y Técnica de la UNNE, que se llevan a cabo en el ámbito de la Facultad de Ciencias Económicas.

idecastro@eco.unne.edu.ar 